\title{
NUTRITIONAL NEEDS IN THE OLDER ADULT, GUIDELINES AND PREVENTION STRATEGIES TO OPTIMIZE HEALTH AND AVOID CHRONIC DISEASE
}

\section{Requisitos nutricionais do idoso, diretrizes e estratégias de prevenção para otimizar a saúde e evitar doenças crônicas}

\author{
Daria Beth Napierkowski ${ }^{\circledR}$, Kimberly Buff Prado ${ }^{b}$ (])
}

Nutritional deficiencies can lead to dependence, decreased functional capacity, frailty, and antisocial behavior. Communitydwelling older adults, especially women and those over ninety years of age, risk malnutrition and, frequently, diminished quality of life. ${ }^{1}$

Diet comprises an important interventional method that decreases the rate of decline for older adults. Multiple studies have shown the association between changed dietary habits and cardiovascular disease and mortality. A diet composed of increased consumption of sugar, refined grains, trans fats/partially hydrogenated oils, cured meats, and sweetened beverages is a significant contributor to inflammation, with subsequent consequences leading to increased mortality. ${ }^{2}$

Time-restricted eating without deprivation of nutrient-dense foods has been studied to assess whether it delays the effects of aging. Longo recommends a series of changes in dietary composition called the Fasting Mimicking Diet, a nutrient-dense, time-restricted diet which can combat inflammation and enhance healthy aging. ${ }^{3}$ Randomized trials have correlated the longevity diet with reduction of risk for cancer, cardiovascular disease, improved glycemic control, and it has been found to impact the production of IGF-1, a growth factor associated with the development of cancer cells. ${ }^{3}$ This controlled dietary change benefits by boosting the body's immune system and decreasing incidence of tumors and inflammatory diseases. ${ }^{4}$

Time-restricted eating in young, non-obese adults has been shown to reduce cardiometabolic risk factors, with the potential for long-term health benefits. ${ }^{5}$ When followed from young adulthood, this lifestyle has clear implications for decreasing chronic illness later in life. Caloric restriction has also been shown to have a slightly positive effect on working memory and could be a key to treating cognitive deficits. ${ }^{6}$ It is important to stress that although caloric restriction has promising results, aging adults must maintain an intake of essential nutrients.

The longevity diet is predominantly plant-based, minimizing consumption of animal-source proteins. Recommendations include consuming healthy sources of protein derived from legumes, chickpeas, green peas, nuts, fish, and eggs; choosing anti-inflammatory foods high in omega fatty acids, such as nuts, flaxseed, or fish; and foods that are nutrient-dense, including vitamin B12 sources such as enriched salmon, anchovies, sardines, cod, shrimp, and clams. ${ }^{3}$ Multiple evidence-based studies

Correspondence data: Daria Beth Napierkowski - William Paterson University - 300 Pompton Road - 07470 - Wayne (NJ), United States. E-mail: darianapierkowski@gmail.com

Received on: 12/29/2020. Accepted on: 05/03/2021

How to cite this article: Napierkowski DB, Prado K. Nutritional needs in the older adult, guidelines and prevention strategies to optimize health and avoid chronic disease. Geriatr Gerontol Aging. 2021;15:e0210027. https://doi.org/10.5327/Z2447-212320212100010

https://doi.org/10.5327/22447-212320212100010 
suggest that the Mediterranean diet, enriched with olive oil, vegetables, fruits, and plant-based proteins, lowers mortality risk. ${ }^{7}$ The Adventist Health Study 2 cohort also showed that consuming plant-based protein in place of meat provided a $40 \%$ reduction in mortality.

Older adults are encouraged to prevent additional weight gain and reduce the risk of chronic disease and disabilities. Some studies recommend time-restricted consumption of two meals within 12 hours, enabling the body to combat inflammation and improve glycemic control. ${ }^{3}$ Improved glycemic control is crucial, since daily consumption of greater than $10 \%$ added sugars were associated with an increased mortality rate. ${ }^{9}$ Dietary patterns of increased consumption of sugar with refined grains correlated with the development of type 2 diabetes, and obesity incurred an increased risk of atherosclerotic cardiovascular disease by $20 \% .{ }^{9}$

Consuming nutrient-dense food has been associated with a lower risk of frailty in community-dwelling older adults. The essential nutrients for older adults are calcium, vitamin $\mathrm{D}$, vitamin B12, fiber, and potassium..$^{10}$ As the body ages, the ability to absorb vitamins often declines. Supplemental vitamins attempt to compensate. However, estimates reveal that less than half of the older adult population utilizes vitamins, given their sociodemographic attitude toward supplementation use. ${ }^{11}$ Aging skin affects the body's absorption of vitamin D from sunshine, affecting the body's ability to absorb calcium. Both calcium and vitamin D supplements are essential in preventing osteoporosis. Water composition decreases with age and with medications, such as diuretics, which can easily lead an older adult to dehydration. The sense of thirst can also decrease with aging. Dehydration can cause the individual to feel tired and enhances the reduction of saliva production. When dehydrated, the older adult often has a dry mouth and succumbs to dysphagia, having difficulty swallowing. Many older adults do not recognize this and instead blame fatigue on "aging." If left untreated, dehydration worsens and can lead to subsequent fatigue, confusion, dizziness, and increased risk of falls.

Adults living in the community are at an increased risk of poor nutrition, especially after age 85 . Factors influencing food intake include oral health, taste changes, medications taken, anorexia, depression, and comorbidities. ${ }^{12}$ Routine screening of older adults for nutrition and physical performance is essential to identifying dietary deficiencies. ${ }^{12}$ Factors influencing malnutrition in older adults include being unmarried, female sex, lack of social resources, and poor physical quality of life. These factors are determinants for the risk of malnutrition. Healthcare providers must also perform a complete oral examination for assessment of broken teeth, swollen gums, ill-fitting dentures, and xerostomia, which might lead to lack of eating or poor eating habits. The number of teeth can impact the risk of malnutrition, although dentures do not always influence food intake.

One of the best tools to assess adults over 65 is the MiniNutritional Assessment Short Form (MNA $\left.{ }^{\circledR}-\mathrm{SF}\right)$. This tool evaluates food intake, weight loss, mobility, acute disease or psychological stress, dementia, depression, and body mass index. ${ }^{13}$

Office visits should include measurement of weight and assessment of body shape, both of which are influenced by genetics and lifestyle. The "apple" body shape with fat primarily in the abdominal area, also known as android obesity, versus pear-shaped (gynoid) obesity, and fat, especially in the upper legs, can help determine the risk of chronic illness. ${ }^{14}$ Android obesity poses a higher cardiovascular risk. Measurements of waist circumference, waist-to-hip ratio, and body mass index are essential assessments. Waist circumference measurement assesses the individual's visceral fat and truncal obesity. If greater than 35 inches in women or 40 inches in men, it can denote an increased risk of metabolic syndrome and cardiovascular disease.

The Nurses'Health study, one of the most extensive reviews conducted to date, studied the impact of waist and hip size measurements on heart disease and cancer in 44,000 volunteer women. After 16 years, women who had a waist size greater than 35 inches or higher had double the risk of dying from heart disease than women with a waist size of fewer than 28 inches. ${ }^{14}$

Challenges to intervention include patient denial of risk of malnutrition, lack of interest, cost of dietetic interventions, and refusal to change dietary habits. To develop a program, the healthcare provider must consider the best method to present material to the older adult. The cost and benefits must be related appealingly to help the client incorporate nutrient-dense foods into their diet. Dietary concerns must be presented with consideration for the adult learner, and an assessment of knowledge of foods can be the first step. Clients should receive written material with explanations of dietary needs. Interventions should include discussing the presence of fruits and vegetables in the diet; specifically, eating three portions of fruit and two portions of vegetables each day will help reduce the risk of frailty. An older adult with complaints of weakness, or decreased exercise tolerance, and unintentional weight loss might indicate frailty syndrome. Interventions can include giving the client a paper prescription with a few dietary suggestions, as this promotes the seriousness of the treatment. Advice should include shopping the periphery of the food market to find fruits and vegetables. The older adult's caregiver should always be included in the discussion of nutrition. Additionally, a referral to 
physical therapy to teach the older adult simple exercises will promote muscle growth and increased strength. An individual exercise program with a nutrient dense diet will help prevent frailty syndrome.

Nutritional education programs can significantly reduce the risk of malnutrition for older adults. Educational interventions designed for older adults can reduce the risk of malnutrition for older dependent adults and improve nutritional dietary intake. ${ }^{15}$ The participation of caregivers in nutritional education is essential, as shopping for food and cooking is often difficult for older adults. Nurse-led educational sessions for community-dwelling older adults have been found to be effective in reducing malnutrition. A one-hour academic session that included caregivers discussed food choices, protein, vitamins, minerals, and water intake, as well as basic cooking skills. ${ }^{15}$ Patients were monitored at home every month for 12 months, and caregivers discussed diet and cooking techniques. ${ }^{15}$ Barthel scores assessing functional independence and BMI were evaluated, and $35.4 \%$ of the control group were found to be malnourished at 12 months of follow-up, while only $4.8 \%$ were malnourished in the intervention group. ${ }^{15}$ Healthcare providers working with community-dwelling adults should include nutritional assessment and education at every visit. The caregiver who shops and helps with the preparation of meals should be included in all educational meetings. Evidence exists that supporting family caregivers as part of the nutrition care team can help prevent malnutrition. Telehealth interventions are one example that can result in increased quality of life as well as improved dietary intake, and are cost-effective. ${ }^{16}$

Older adults are at increased risk for imbalances in nutritional status which can be impacted by cognitive as well as social factors, such as depression, isolation, dementia, or limited income. By assessing the individual's nutritional status through screening and assessment and intervening with treatment and education, the healthcare provider can develop a care plan that optimizes the older adult's health. Older adults have a fixed income and often shop at several stores to find prices that fit their food budget. Environmental factors that influence food choices include transportation to places to shop and the types of food available. Essential considerations include functional limitations of the older adult and cultural references when discussing food choices. Older adults will make better food choices when nutritious foods are available and convenient in their neighborhood. A variety of nutritional educational resources can influence dietary choices and positively impact the nutritional status of the older adult, and possibly prevent malnutrition.

\section{CONFLICTS OF INTEREST}

The authors declare no conflicts of interest.

\section{FUNDING}

This research did not receive any specific grant from funding agencies in the public, commercial, or not-for-profit sectors.

\section{AUTHORS' CONTRIBUTION}

DBN: conceptualization, investigation, writing - original draft, writing - review \& editing. KBP: conceptualization, investigation, writing — original draft, writing review \& editing.

\section{REFERENCES}

1. Hernandez-Galiot A, Goni I. Quality of life and risk of malnutrition in a home dwelling population over 75 years old. Nutrition. 2016;35:81-6. https://doi.org/10.1016/j.nut.2016.10.013

2. Wilson MM, Reedy J, Krebs-Smith S. American diet quality: Where it is, where it is heading, and what it could be. J Acad Nutr Diet. 2016;116(2):302-10.e1. https://doi.org/10.1016/j.jand.2015.09.020

3. Longo V. 2019. Longevity diet for adults. Available at: https://valterlongo. com/daily-longevity-diet-for-adults/. Accessed on: Mar. 2021.

4. Wei M, Brandhorst S, Shelehchi M, Mirzaei H, Cheng CW, Budniak J, et al. Fasting-mimicking diet and markers/ risk factors for aging, diabetes, cancer, and cardiovascular disease. Sci Transl Med. 2017;9(377):eaai8700. https://doi.org/10.1126/scitranslmed.aai8700

5. Kraus W, Bhapkar M, Huffman K, Pieper CF, Das SK, Redman LM, et al. 2 years of calorie restriction and cardiometabolic risk (CALERIE); Exploratory outcomes of a multicenter, phase 2, randomized controlled trial. Lancet Diabetes Endocrinol. 2019;7(9):673-83. https://doi.org/10.1016/s2213-8587(19)30151-2
6. Leclerc E, Trevizol AP, Grigolon AB, Subramaniapillai M, Mclntyre $\mathrm{RS}$, Brietzke $E$, et al. The effect of caloric restriction on working memory in healthy non-obese adults. CNS Spectr. 2020;25(1):2-8. https://doi.org/10.1017/s1092852918001566

7. Estruch R, Ros E, Salas-Salvadó J, Covas M-I, Corella D, Arós F, et al. Primary prevention of cardiovascular disease with a Mediterranean diet supplemented with extra-virgin olive oil or nuts. N Engl J Med. 2018;378(25):e34. https://doi.org/10.1056/nejmoa1800389

8. Tharrey M, Mariotti F, Mashchak A, Barbillon P, Delattre M, Fraser GE. Patterns of plant and animal protein intake are strongly associated with cardiovascular mortality: the Adventist Health study-2 cohort. Int J Epidemiol. 2018;47(5):1603-12. https://doi. org/10.1093/ije/dyy030

9. Yang Q, Zhang Z, Gregg EW, Flanders WD, Merritt R, Hu FB. Added sugar intake and cardiovascular disease mortality among US adults. JAMA Inter Med. 2014;174(4):516-24. https://doi.org/10.1001/ jamainternmed.2013.13563 
10. Shlisky J, Bloom D, Beaudreault AR, Tucker KL, Keller HH, Freund-Levi $Y$, et al. Nutritional considerations for health aging and reduction in age related chronic diseases. Adv Nutr. 2017;8(1):17-24. https:// dx.doi.org/10.3945\%2Fan.116.013474

11. Sebastian RS, Cleveland LE, Goldman JD, Moshfegh AJ. Older adults who use vitamin/mineral supplements differ from nonusers in nutrient intake adequacy and dietary attitudes. J Am Diet Assoc. 2007;107(8):1322-32. https://doi.org/10.1016/j.jada.2007.05.010

12. Chatindiara I, Williams V, Sycamore E, Richter M, Allen J, Wham C. Associations between nutrition risk status, body composition, and physical performance among community dwelling older adults. Aust N ZJ Public Health. 2019;43(1):56-62. https://doi.org/10.1111/1753-6405.12848

13. Kaiser M, Bauer J, Ramsch C, Uter W, Guigoz Y, Cederholm T, et al. Validation of the mini nutritional assessment short form (MNA ${ }^{\circledR}$-SF): A practical tool for identification of nutritional status. J Nutr
Health Aging. 2009;13(9):782-8. https://doi.org/10.1007/s12603009-0214-7

14. Harvard T.H. Chan, School of Public Health. Waist size Matters, How abdominal fat increase disease risk. 2020. Available at: https://www. hsph.harvard.edu/obesity-prevention-source/obesity-definition/ abdominal-obesity/. Accessed on: Mar. 2021.

15. Fernández-Barrés S, García-Barco M, Basora J, Martínez T, Pedret $\mathrm{R}$, Arija $\mathrm{V}$, et al. The efficacy of a nutrition education intervention to prevent risk of malnutrition for dependent elderly patients receiving home care: A randomized controlled trial. Int J Nurs Stud. 2017;70:131-41. https://doi.org/10.1016/j.ijnurstu.2017.02.020

16. Marshall S, Agarwall E, Young A, Isenring E. Role of domiciliary and family carers in individualized nutrition support for older adults living in the community. Maturitas. 2017:98:20-9. https://doi.org/10.1016/j. maturitas.2017.01.004 